\title{
Patient encounters in very deprived areas
}

Early in the Deep End project, 15 GPs met for a workshop on patient encounters in very deprived areas, drawing on experience, evidence, and policy, and focusing on what can be achieved and how.

Consultations with patients are the largest and most important part of the work of GPs. In severely deprived areas, consultations are characterised by multiple morbidity (including psychological and social problems), reduced expectations, time constraints, lower patient enablement, and practitioner stress. ${ }^{1}$

Consultations always address the problems presented by patients on the day (reactive care), but can also address potential future problems (anticipatory care). A key aspect of the consultation is the relationship between the patient and the doctor, who often know each other from previous consultations. This prior experience is an important aspect of the professional intuition required to know how and when to extend the aims of a consultation. Maintaining the relationship and ending on a positive note are important outcomes of each consultation.

Research has shown that patients in deprived areas are less likely than patients in affluent areas to wish to have an active role in decisions concerning their care. ${ }^{2}$ Patients may also be less interested and ready to address changes in health behaviour. Addressing such issues within consultations is time-consuming and is often not immediately effective. Explanations may take longer due to problems in health literacy. Practitioners describe 'chipping away' at these issues, rather than achieving large and sudden changes in behaviour. ${ }^{3}$

Whether a consultation includes more than reactive care depends on many factors, including appropriateness, having time available, and patient and practitioner expectations. Practitioner stress can affect both practitioner and patient behaviour within a consultation, influencing what the patient presents and how the practitioner responds. $^{3}$ NHS policies tend to underestimate the constraints and difficulties in moving beyond reactive patterns of patient and practitioner behaviour.

The incentives of the Quality and Outcomes Framework do not reward practitioners for extending consultations beyond a narrow range of targets and the QOF agenda, highlighted via computer alerts, can be felt as an intrusion in the consultation. Current NHS initiatives concerning patient self-help and selfmanagement appear to have poor penetration in deprived areas and were not recognised by practitioners at the workshop.

Consultations are more likely to be successful if carried out in a systematic way, establishing the patient's agenda at the outset, picking up clues ('psycho-social red flags') and ending with clear agreement as to what has been decided. Surgeries (serial consultations) can be made more efficient by good practice organisation, involving clear communication and the involvement of other members of the team including receptionists and practice nurses.

A frequent and important aspect of many consultations is referral to other professionals and services. Referral is most likely to be taken up when it is quick and to a familiar local setting. When a referral is made outside the practice, some patients would benefit from additional help and reminders to increase the probability of the referral being taken up.

Practices provide a hub for referral to a huge range of other professions and services. Many of these pathways are dysfunctional, with poor communication and feedback. Multiprofessional working across organisational boundaries works best via established relationships with named individuals, with regular, reliable contact and opportunities for professional exchange.

Practitioners are keen to make use of the full range of possible services and sources of help for patients but frequently lack accurate and up-to-date information about what is available locally. Patients also need ready access to health information and resources available within the local

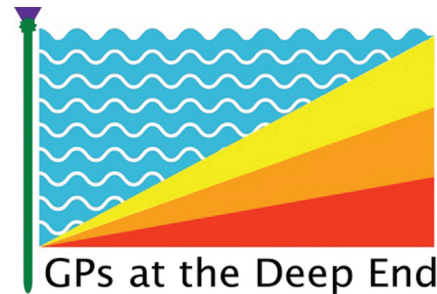

community. It is not clear whose job it is to provide such information and to keep it current.

Evidence is needed on the best ways of providing access to different types of consultation (simple or complex), using additional time within consultations, linking with other professional colleagues and services, making good use of community information, and providing support for both patients and professionals.

There are too few opportunities for practitioners working in severely deprived areas to share experiences and views concerning the conduct of consultations and the organisation of practices. Additional education and training is required not only for young practitioners preparing to work in deprived areas, but also for established practitioners, building on their substantial knowledge, experience, and ideas to improve services for patients.

The most important barrier to addressing the inverse care law remains the shortage of time within consultations. Unless this root cause of the inverse care law is addressed, the resulting inequalities in health will persist. ${ }^{4}$ It is encouraging that Members of Parliament serving deprived areas in Scotland and in England are beginning to bang this drum.

\section{Graham Watt}

On behalf of the Deep End Steering Group.

\section{REFERENCES}

1. Mercer SW, Watt GCM. The inverse care law: clinical primary care encounters in deprived and affluent areas of Scotland. Ann Fam Med 2007; 5: 503-510.

2. McKinstry B. Do patients wish to be involved in decision making in the consultation? A cross-sectional survey with video vignettes. $B M J$ 2000; 321: 867-871.

3. GPs at the Deep End. Deep End report 6. Patient encounters in very deprived areas; what can be achieved and how? The sixth meeting of 'GPs at the deep end', 14 May 2010. http://www.gla.ac.uk/media/media_153244_en.pdf (accessed 10 Jan 2010).

4. RCGP Scotland Health Inequalities Short Life Working Group. Time to care. Heath inequalities, deprivation and general practice in Scotland. Edinburgh: RCGP Scotland, 2010.

DOI: 10.3399/bjgp11X556380 